Open Peer Review on Qeios

\title{
Socioeconomic and behavioural determinants of oral health in 6- and 7-year-old schoolchildren from Palermo (Sicily)
}

Simonetta d'Alba ${ }^{1}$, Giovanna Giuliana ${ }^{1}$, Giuseppe Pizzo $^{1}$, Domenica Matranga ${ }^{1}$, Anna Maria Maltese ${ }^{1}$, Fortunato Buttacavoli ${ }^{1}$

1 University of Palermo

Funding: The author(s) received no specific funding for this work.

Potential competing interests: The author(s) declared that no potential competing interests exist.

\section{Abstract}

Caries and periodontal diseases are highly neglected disease affecting millions of Italian children; both diseases are preventable and the combination of high prevalence, high morbidity and a relative lack of attention of the Italian National Health Service make caries and periodontal disease a significant public health problem.

To date none studies reported epidemiological data of 6-7-year-old-italian-children-population.

The aim of this survey was to describe the dental and periodontal health status in 6- and 7-year-old schoolchildren from Palermo and to evaluate the association between socioeconomic determinants and behavioural habits.

A sample of 2701 children aged 6 and 7 from the selected public schools in all the eight districts of the town received a clinical intraoral examination carried out by trained dentists. Parents were asked to fill in a questionnaire investigating children's eating habits, oral health related behaviours and socioeconomic determinants of the household.

The percentage of 6- and 7-year-old schoolchildren with at least one carious lesion was found to be $66.5 \%$. A higher mother's and father's educational level were a protective factor for carious lesions; conversely, a paternal unemployment was found to be a risk factor. Living in II and VII districts was a risk factor for all stages of carious lesions.

Key words: Dental caries; Schoolchildren; Oral health-related determinants; Caries risk factors; ICDAS

II; Caries epidemiology; Deprivation index; Oral health promotion; Dental sealants; Di.Chir.On.S 2021

\section{Introduction}

Caries and periodontal diseases are highly neglected disease affecting millions of Italian children; both diseases are preventable and the combination of high prevalence, high morbidity and a relative lack of attention of the Italian National Health Service make caries and periodontal disease a significant public 
health problem.

To date few epidemiological surveys were conducted with the regard to the oral health of the Italian children population and namely three of these are based on DMFT-dmft index ${ }^{[1]}(2)$; a recent national survey (1) ${ }^{[2]}$ is based on ICDAS-II method, but it investigated 12-year-old children. Moreover, one work investigated the prevalence of caries of schoolchildren living in Palermo, but none of the previous studies reported data of 6-7-year-old-children. Since the eruption of the first permanent molar (FPM) occurs during the $6^{\text {th }}$ and $7^{\text {th }}$ years, in this critical period the FPM's carious involvement could already occur. In this perspective, the World Health Organization (WHO) and the University of Milan have promoted a national epidemiological study aimed at a sample of 4, 6 and 12-years-old students from schools in the North, Central and South Italy. The aim of this survey was to describe the dental and periodontal health status in 6- and 7-year-old schoolchildren from Palermo and to evaluate the association between socioeconomic determinants and behavioral habits.

\section{Material and Methods}

Children aged 6 and 7 from the selected public schools in all the eight districts of the town received a clinical intraoral examination by trained dentists. The data collection of this study was carried out from February 2017 to February 2019 to detect dental and periodontal status in children resident in the city of Palermo (age groups: 4-5, 6-7 and 12 years).

The schools enrolled in the survey were selected on the basis of the geographical location in the 8 districts of Palermo (Figure 1) and according to the school headmasters' willingness and availability to conduct the survey.

An e-mail explaining the nature of the survey was sent to the headmasters of the selected schools with the aim of inviting them to collaborate with the survey.

A letter explaining the nature of the survey and containing a pre-printed form of informed consent of participation to the survey was delivered by the schools to parents/caregivers of the subjects to be examined. Children were enrolled as participants only with the consent properly signed.

Carious lesions were classified according to the modified International Caries Detection Assessment System (ICDAS-II) $(3,4)^{[3][4]}$; the Community Periodontal Index (CPI) was used for periodontal assessment. Parents were asked to fill in a questionnaire investigating children's eating habits, oral health related behaviours and socioeconomic determinants of the household.

The sample number for each single age group has been identified with a 99\% confidence level; and each group was increased by $25 \%$ to counterbalance the effect of any non-responders (persons who were not examined or who did not fill in the questionnaire properly). 


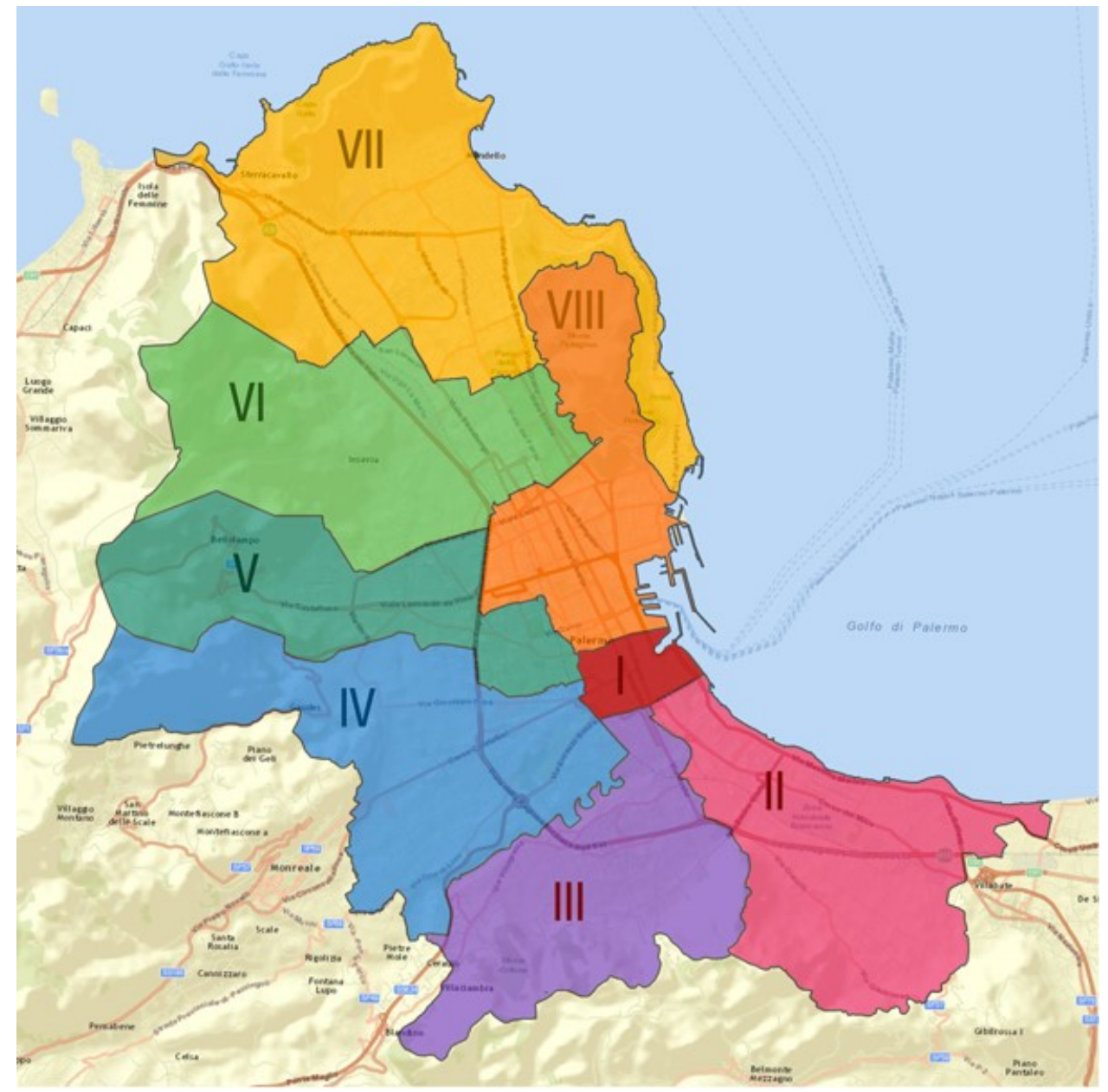

Figure 1. Municipal districts of Palermo.

\section{Results}

During the study period, oral health data were collected from a total of 2701 subjects (males: 1327/49.13\%; females: 1374/50.87\%); of these 946 were 3-5 years old, 995 were 6-7 years old and 760 were 12-13 years old. The percentage of 6- and 7-year-old schoolchildren with at least one carious lesion was found to be $66.5 \%$. A higher mother's and father's educational level were a protective factor for carious lesions; conversely, a paternal unemployment was found to be a risk factor. Children living in II and VII districts (strictly related to poor sociodemographic status) were a risk factor for all stages of carious lesions. Frequent sweet drinks and chewing gum consumption was significantly associated with caries. The percentage of children with untreated caries was on FPMs $26.5 \%$ and of these only $6.1 \%$ had restorations.

\section{Conclusions}

This survey demonstrates that occurrence of carious lesions in 6- and 7-year-old schoolchildren is quite high, with $662(66,5 \%)$ children had at least one lesion, whereas only 60 (9.1\%) of these have restorations. Therefore, only a small part of the sample visited a dentist for regular check-ups, while the most of carious lesions remained untreated and the treatment need was unacknowledged.

Oral health and risk of caries is significantly associated with socioeconomic factors and behavioural 
determinants. ${ }^{[5]}$ The most underprivileged and poor city neighbourhoods were related to higher levels and risk of caries. The frequency of sweets drinks and chewing gum is a risk factor for caries development. ${ }^{[6][7]}$ There was a very low prevalence of fissure sealants on FPMs, indicating the underutilization of fissure sealants among the schoolchildren examined in this study. School-based or national sealant programs should be promoted intensely, implemented as a successful preventive approach and completed with oral health education.

\section{References}

1. ^Italo Francesco Angelillo, Rosa Anfosso, Carmelo Giuseppe A. Nobile, Maria Pavia. (1998). European Lournal of Epidemiology. doi:10.1023/a:1007471707836.

2. `Guglielmo Campus, Fabio Cocco, Laura Strohmenger, Maria Grazia Cagetti. (2020). Caries severity and socioeconomic inequalities in a nationwide setting: data from the Italian National pathfinder in 12-years children. Sci Rep, vol. 10 (1). doi:10.1038/s41598-020-72403-x.

3. A A. I. Ismail, W. Sohn, M. Tellez, A. Amaya, et al. (2007). The International Caries Detection and Assessment System (ICDAS): an integrated system for measuring dental caries. Commun Dent Oral Epidemiol, vol. 35 (3), 170-178. doi:10.1111/j.1600-0528.2007.00347.x.

4. `NB Pitts, KR Ekstrand, The ICDAS Foundation. (2013). International Caries Detection and Assessment System (ICDAS) and its International Caries Classification and Management System (ICCMS) - methods for staging of the caries process and enabling dentists to manage caries. Community Dent Oral Epidemiol, vol. 41 (1), e41-e52. doi:10.1111/cdoe.12025.

5. `Mariantonietta Arrica, Giovanna Carta, Fabio Cocco, Maria Grazia Cagetti, et al. (2017). Does a social/behavioural gradient in dental health exist among adults? A cross-sectional study.J Int Med Res, vol. 45 (2), 451-461. doi:10.1177/0300060516675682.

6. `Melvin B. Heyman, Steven A. Abrams, Leo A. Heitlinger, Michael deCastro Cabana, et al. (2017). Fruit Juice in Infants, Children, and Adolescents: Current Recommendations. doi:10.1542/peds.2017-0967.

7. `Paula Moynihan, Poul Erik Petersen. (2004). Diet, nutrition and the prevention of dental diseases. Public Health Nutr., vol. 7 (1a), 201-226. doi:10.1079/phn2003589. 